www.jmscr.igmpublication.org Impact Factor 5.84

Index Copernicus Value: 83.27

ISSN (e)-2347-176x ISSN (p) 2455-0450

crossref DOI: _https://dx.doi.org/10.18535/jmscr/v5i4.42

Journal Of Medical Science And Clinical Research

\title{
Significance of Brainstem Auditory Evoked Potential in Cerebral Palsy
}

\author{
Authors
}

\section{Sreejith Kalathummarathu ${ }^{1}$, Shehadad Kammili ${ }^{2}$, Mohanraj manjalavil ${ }^{3}$ Biju Gopinath ${ }^{4}$ P. Sreedevi Menon ${ }^{5}$, George Joseph.N ${ }^{6}$}

${ }^{1}$ Assistant Professor, Dept of Physical Medicine and Rehabilitation, Govt Medical College, Kozhikode

${ }^{2}$ Assistant Professor, Dept of Physical Medicine and Rehabilitation, Govt Medical College, Kozhikode

Email: drshehadad@gmail.com,Ph: 9946909044

${ }^{3}$ Assistant Professor, Dept of Physical Medicine and Rehabilitation, Govt Medical College, Kozhikode

Email: rajmanjalavil@yahoo.co.in,Ph: 9447478276

${ }^{4}$ Consultant Physiatrist, Physical Medicine and Rehabilitaion Hospital, Ministry of Health, Kuwait

Email: bijugopi86@gmail.com, Ph:+96525625890

${ }^{5}$ Professor, Dept of Physical Medicine and Rehabilitation, Govt Medical College, Kozhikode

Email: devisreemukund@yahoo.com,Ph: 9947412900

${ }^{6}$ Professor, Amritha Institute of Medical Sciences, Kochi

Email-georjosen53@gmail.com,Ph: 9447071253

Corresponding Author

\section{Sreejith Kalathummarathu}

Assistant Professor, Dept of Physical Medicine and Rehabilitation, Govt Medical College, Kozhikode.

Email: sreejithpmr@gmail.com Ph: 8547184205

\begin{abstract}
Background: Cerebral palsy is one of the most common disabilities affecting the children. The early detection and accurate investigations is the key for an early intervention to minimise the long lasting sequelae. Brainstem Auditory Evoked Potential (BAEP) is a neurophysiologic technique used to assist in measuring hearing thresholds in infants at risk for hearing loss and to assess the brainstem auditory pathways. It records a series of potentials corresponding to the sequential activation of peripheral, pontomeduallary, pontine, and midbrain portions of the auditory pathways. BAEP if abnormal is found to be predictive of neurod evelopmental delay and abnormalities of hearing and language in pediatric population.

Aim: To study BAEP profile in cerebral palsy $(C P)$ and look for any correlation with the severity and type of disease.

Materials and Methods: BAEP was perfomed in 21 children with $C P$ in the age range of 1-2 after performing a detailed neurological evaluation.

Results: Though there were many abnormal cases of BAEP statistically significant change was not obtained with any variable. There was no correlation of BAEP with clinical severity also.

Conclusion: BAEP abnormalities are not consistent in children with CP. The abnormalities are not related to the severity of the disease also.

Key Words: Brainstem Auditory Evoked Potential, Cerebral Palsy.
\end{abstract}




\section{Introduction}

Cerebral palsy is one of the most common disabilities affecting children. The reported incidence varies but is approximately 2to3per 1000 live births ${ }^{1}$. Brain injury that leads to $\mathrm{CP}$ can occur in the prenatal, perinatal or postnatal period $^{2}$. The combination of immaturity, fragile brain vasculature, and the physical stresses of immaturity combine to predispose these children to compromised cerebral blood flow ${ }^{3}$ and can be of varying severity according to the size and extent of cerebral micro vascular haemorrhage. Very low birth infants have increased incidence of periventricular hemorrhagic infarction which upon healing produces the characteristic periventricular leucomalacia ${ }^{4}$. The post natal causes of $\mathrm{CP}$ can include almost any kinds of brain injury. Most common perinatal injuries are severe anoxic or ischemic brain injury.

Depending upon the type of involvement CP can be spastic, dystonic, athetoid and hypotonic or mixed. It can be classified depending on the extent of body involvement as monoplegia, hemiplegia, diplegia or quadriplegia 5 .

The most striking difficulties associated with $\mathrm{CP}$ are disorders of neuromuscular control. In infancy various manifestations like irritability, lethargy, weak suck with tongue thrust, poor head control etc. occurs. Motor delay can also be suggested by persistent abnormal motor activities, such as rolling for mobility, bunny hopping, or hand preference before first birthday ${ }^{6}$. Abnormal postures like scissoring occurs as a result of abnormal tone and weakness of specific muscles. Some abnormal postures are as a result of persistence of primitive reflexes like asymmetric tonic neck reflex. Dyskinetic and ataxic movement patterns are hallmark of certain subtypes of CP.

The most common associated abnormality in $\mathrm{CP}$ is abnormality of occulomotor system. A large number of children with CP has abnormalities of oromotor function. Mental retardation is found in $30 \%$ of children with $\mathrm{CP}$ and seizures are found in a third of affected children ${ }^{6}$. Hearing disabilities are rare in $\mathrm{CP}$ and when present they are associated with congenital infections. Neonatal hyperbilirubinemia is a cause of sensoryneural deafness.

\section{Brainstem Auditory evoked potential (BAEP)}

BAEP is a neurophysiologic technique used to assist in measuring hearing thresholds in infants at risk for hearing loss and in nonverbal children. Hearing impairment early in life leads to abnormal speech and language development. Early identification of hearing impairment can allow for intervention. Those newborn infants considered at risk of hearing loss should be tested and they include infants with a family history of deafness, congential infections, prematurity, CNS malformations, perinatal asphyxia and hyper bilirubinemia. Approximately 1- 5\% of children leaving a neonatal intensive care unit have bilateral sensorineural hearing loss requiring amplification. Care should be taken when assessing auditory threshold in children with neurologic disorders producing abnormal wave forms. Serial BAEPs should be done to assess whether there is improvement before prescribing amplification.

BAEP provides a very useful tool in paediatric patients who may not be able to communicate. The study can be used as an adjunct to the clinical examination. The BAEP is a useful method of evaluating a variety of pathologic conditions that can damage the brainstem auditoy pathways ${ }^{7}$. After an appropriate acoustic stimulus a series of potentials corresponding to the sequential activetion of peripheral, pontomeduallary, pontine, and midbrain portions of the auditory pathways are obtained in the first 10 milliseconds. The latencies between these potentials reflect the neural conductions in the corresponding segments of the central auditory pathway. The stimulus is a square wave pulse of $0.1 \mathrm{~ms}$ duration. This can either move the diaphragm towards the ear (condensation) or away from the ear (rarefaction).

BAEP is normal in cortical deafness and gross BAEP abnormalities are ofen accompanied by completely normal behavioural parameters. 


\section{Effect of drugs}

Interpeak latencies are not affected by therapeutic doses of CNS depressant drugs.

BAEP recorded in anoxic brain damage is often abnormal and may be helpful in predicting long term neurological sequelae in children after asphyxia. BAEP is used to identify infants at risk of neurological sequelae after hypoxic brain injury.

\section{Review of Literature}

Stockyard et al. (1983) found there was no consistent relationship between BEAP finding and severe brain damage. Majnemer et al. in their study found out that abnormal BAEP in neonates are predictive of gross motor delay and abnormal neurological examination at age $1^{8}$. The abnormalities included increased IPLs, abnormal wave5/wave1 amplitude ratios and abnormal wave morphology. Roberson JB etal. ${ }^{9}$ compared BAEP and auditory steady state response in children from 7-61 months and concluded that ASSR showed sensitivity equal to BAEP in hearing level from $0-90 \mathrm{~dB}$ and in hearing impairment greater than $90 \mathrm{~dB}$ normal hearing level ASSR showed distinct advantage over ABR.

Study conducted in department of developmental disorders, National Institute of mental health, Kohonodaie, Ichikawa, Japan evaluated Otoacousitc emission (OAE) with BEAP and concluded that careful examination of both tests might be necessary for further assessment of the hearing function in pediatric patients with neurological disorders and specific auditory nerve disease. Chadha S \&BAIS as conducted baep study in $\mathbf{5 0}$ high risk neonates in ICU and compared with normal controls. They found out that prolonged latency in wave 5 and wave 1-5 interwave interval prolongation in the study group with a significant auditory impairment of $18 \%{ }^{10}$. Morlet Tetal. conducted hearing screening test in high risk neonates $b$ transient evoked oto acoustic emission followed by BAEP in suspected cases of heaing loss. Their results showed that early hearing loss screening by these modalities in at risk neonates needs to be persued ${ }^{11}$.

Pasnan JW et al. conducted a study of brainstem middle ear latency and cortical auditory evoked responses (BMC-AER) in preterm infants. They concluded that in predicting neurodevelopmental outcome in pre term infants BMC-AER is of limited value ${ }^{12}$. Maison $S$ etal. did a study of BAEP in 72 children showing inadequate language development and found out that $\mathrm{B} / \mathrm{L}$ BAEP abnormalities were associated with language retardation ${ }^{13}$. Stueve MP etal. conducted a study which supported the inclusion of ASSR (Auditory steady state response) in to the standard paediatric test battery in detecting hearing loss ${ }^{14}$.

Mjoen S etal. conducted a study where ABR were recorded in 60 high risk neonates and identifying the non responders helped in early audiological training program for these children ${ }^{15}$.The study conducted with ABR in newborns and infants by Yamada etal. indicated that frequency selective ABR can be applied clinically as an objective method of audiometry for newborns and infants ${ }^{16}$. Tapia Toca MC etal. conducted a study on children with auditory neuropathy. In this study they concluded that auditory multiple steady state response (MSSR) technique is useful as an objective electro audiometric tool in patients with auditory neuropathy as ABR. This technique is a recommendable test to complete the audiological study in infants with auditory neuropathy to establish a more precise treatment ${ }^{17}$.

Luo $\mathrm{R}$ etal. explored the clinical and audiological characterisatics of sensorineural hearing loss with pathological changes both in cochlea and retrocochlear in children and evaluated the relationship between SHL and lesions in the CNS. They concluded that the patients with SHL are usually accompanied with CNS diseases in childhood and their hearing loss appears very different from audiological characteristics. They suggested that it is necessary to test both ABR and DPOAE in these patients ${ }^{18}$.

Bao X etal. conducted BAEP on children with meningitis to assess the incidence of hearing 
impairment. The incidence of hearing loss after bacterial and aseptic meningitis is high. BAEP is useful to screen for possible hearing loss in children with meningitis and follow up. BAEP is necessary for those patients with initial abnormal BAEP $^{19}$.

\section{Materials and methods}

\section{Aim}

To study the BAEP profile in children with cerebral palsy and to analyse whether there is any correlation between disease severity and type with BAEP abnormality.

\section{Study Population}

21 Cerebral palsy children of 1to 2 year of age attending Physical Medicine and Rehabilitation OPD, Medical college, Kozhikode, Kerala during one year period were selected. After initial assessment \&neurological examination BAEP is done from the neurophysiologic lab of Physical Medicine and Rehabilitation department, free of cost.

Inclusion criteria

- $\mathrm{CP}$ children-according to the definition of Mutch and colleagues ${ }^{20}$ with moderate to severe development delay.

Exclusion criteria

- $\mathrm{CP}$ with uncontrollable seizures

- $\mathrm{CP}$ with severe other congenital anomalies

- $\quad$ P with minimal deficits

\section{Performance of BAEP}

In our study surface electrodes filled with conducting jelly were used. Surface electrodes are placed at the vertex and ipsilateral earlobes or mastoids. The electrode impedance is kept below $5 \mathrm{~K}$ Ohms. The ears are stimulated monaurally. If binaural stimulation is used, the normal waveforms generated by the good ear mask the abnormality in the bad ear. The four channel montage for recording BAEPs are

Channel 1: $\quad \mathrm{Ai}-\mathrm{Cz}$

CHANNEL 2: $\mathrm{Ac}-\mathrm{Cz}$

Channel 3: $\mathrm{Ai}-\mathrm{Cz}$

Channel 4: inion-Cz
Ai and Ac refer to ipsi lateral and contra lateral to the earlobe to be stimulated. Usually one channel is essential for clinical interpretation.

Stimulation--: BEAPs are produced by a brief click stimulus through ear phones which is usually a square wave pulse of $0.1 \mathrm{~ms}$ duration. The pulse can move the ear phone diaphragm either towards or away from the ear. The ear phone movement towards the ear is called condensation phase stimulus, whereas the one away from the patient's ear is called rarefaction stimulus. Amplitude of wave 1 ends to be greater with rarefaction clicks compared to condensation clicks. Since the recognition of wave 1 is very important rarefaction polarity is used. In our study a click rate of $31 \mathrm{~Hz}$ is used.

The BEAP are recorded during sleep. The responses recorded from surface electrodes placed on the vertex and the ipsilateral mastoid or ear lobe.

Wave 1 is the prominent initial up going peak in the ipsilateral ear recording channel. It appears $1.4 \mathrm{~ms}$ after the stimulus. Medial ear lobe recording provides higher amplitude of wave 1 compared to mastoid. Wave 1 amplitude may be improved by

1. Increasing the stimulus intensity

2. Decreasing the stimulus rate.

3. Alternating click polarity.

Patients who have only central nervous system problems, should have a preserved wave 1 since it originates from eighth nerve. Conversely the patients with significant peripheral impairment may have a very poorly formed or absent wave 1 but relatively normal other waves.

Wave 2 is poorly formed in some adults and most neonates. It sometimes appears as a small peak along the down going slope of wave 1 or in the up going slope of waved 3 .

Wave 3 is usually a prominent peak and is followed by a prominent trough. In contralateral channel, wave 3 often appears smaller and earlier than the ipsilateral ear. This feature helps in wave 3 recognition. 
Wave 5 is the most prominent peak appearing 5.5 ms after the stimulus. It starts above the baseline and its trough is maximum below the baseline.

Wave $4 \& 5$ may have the following patterns

1. Single peak which is completely fused as a tall wide pyramid.

2. Two peaks which are close but still visibly separated.

3. Wave 4 may be on the up going slope of wave 5 .

4. Wave 5 may be on the down going slope of wave 4.

In this study BAEP is done at $40 \mathrm{~dB}$ NHL, $70 \mathrm{~dB}$ NHL \& 90 dB NHL. dB NHL is a scale relative to normal hearing level for a particular stimulus. This is based on the average hearing threshold for that stimulus of a group of normal hearing subjects. For example with a particular stimulator and stimulus, a group of such subjects might have an average click hearing threshold (the intensity level at which they can barely hear the click ) of $20 \mathrm{~dB}$ peSPL (peak equivalent Sound Pressure Level). The normal hearing threshold is about 30 $\mathrm{dB}$ peSPL. PeSPL is the intensity of a long duration sound that has the same amplitude as the peak SPL of a short duration sound that has the same amplitude as the peak SPL of a short duration sound whose intensity is the measure desired. This is determined by using a sound level meter often with a comparison made to a reference. Zero dbHL is then defined as the normal group's average threshold. If another patient is stimulated at $60 \mathrm{~dB}$ peSPL, when the threshold of the normal was $20 \mathrm{Db}$ peSPL, the stimulus intensity is (60-20) 40Dbnhl. Impedance must be maintained below $5 \mathrm{~K}$ ohms.

BAEPS are best recorded using an amplification of 200,000 to 500,000 . The filters are 50 to $150 \mathrm{~Hz}$ low cut off and $3,000 \mathrm{~Hz}$ high cut off. The average is set for total sweep duration of 10 to $12 \mathrm{msec}$. Each recording should be sampled with a maxim- um inter sample interval of $0.1 \mathrm{msec}$. The automatic sweep repetitions should be set to 1000 . 2000 trails are averaged. Two repetitions are done and superimposed to check for reproducibility. BAEP repetitions should superimpose almost exactly. The latency values measured on two separate repetitions should agree with each other within $0.1 \mathrm{~ms}$ or less. The amplitude values should agree with each other within $10 \%$.

The following parameters are measured for analysis of BAEPs.

1. Absolute latency

2. Inerpeak latencies (IPL)

The BAEP abnormalities may include one or more of the following:

1. Absence of wave forms.

2. Abnormal interpeak latencies.

3. Significant right to left asymmetry.

Tables

Correlation between tone and BAEP

Cross tab at $70 \mathrm{~dB}$ NHL

\begin{tabular}{|l|c|c|c|}
\hline TONE & NORMAL & ABNORMAL & TOTAL \\
\hline HYPO & & 4 & 4 \\
\hline GRADE I & & 2 & 2 \\
\hline GRADE2 & 4 & 4 & 8 \\
\hline GRADE3 & 5 & 2 & 7 \\
\hline TOTAL & 9 & 12 & 21 \\
\hline
\end{tabular}

Chi-Square test

\begin{tabular}{|c|c|c|c|}
\hline & Value & Df & Asymp.Sig(2sided) \\
\hline Pearson Chi-Square & 7.000 & 3 & .072 \\
\hline
\end{tabular}

Crosstab 90 dB NHL

\begin{tabular}{|l|c|c|c|}
\hline TONE & NORMAL & ABNORMAL & TOTAL \\
\hline HYPO & & 4 & 4 \\
\hline GRADEI & & 2 & 2 \\
\hline GRADE II & 3 & 5 & 8 \\
\hline GRADE III & 4 & 3 & 7 \\
\hline TOTAL & 7 & 14 & 21 \\
\hline
\end{tabular}

Chi -Square test

\begin{tabular}{|l|c|c|c|}
\hline & Value & Df & $\begin{array}{c}\text { Asymp.Sig } \\
\text { (2sided) }\end{array}$ \\
\hline Pearson-Chi-Square & 4.848 & 3 & 0.183 \\
\hline
\end{tabular}




\section{JMSCR Vol||05||Issue||04||Page 19985-19992||April}

Fig 1. Normal BAEP showing different waves

AEP RECORD (nHL)
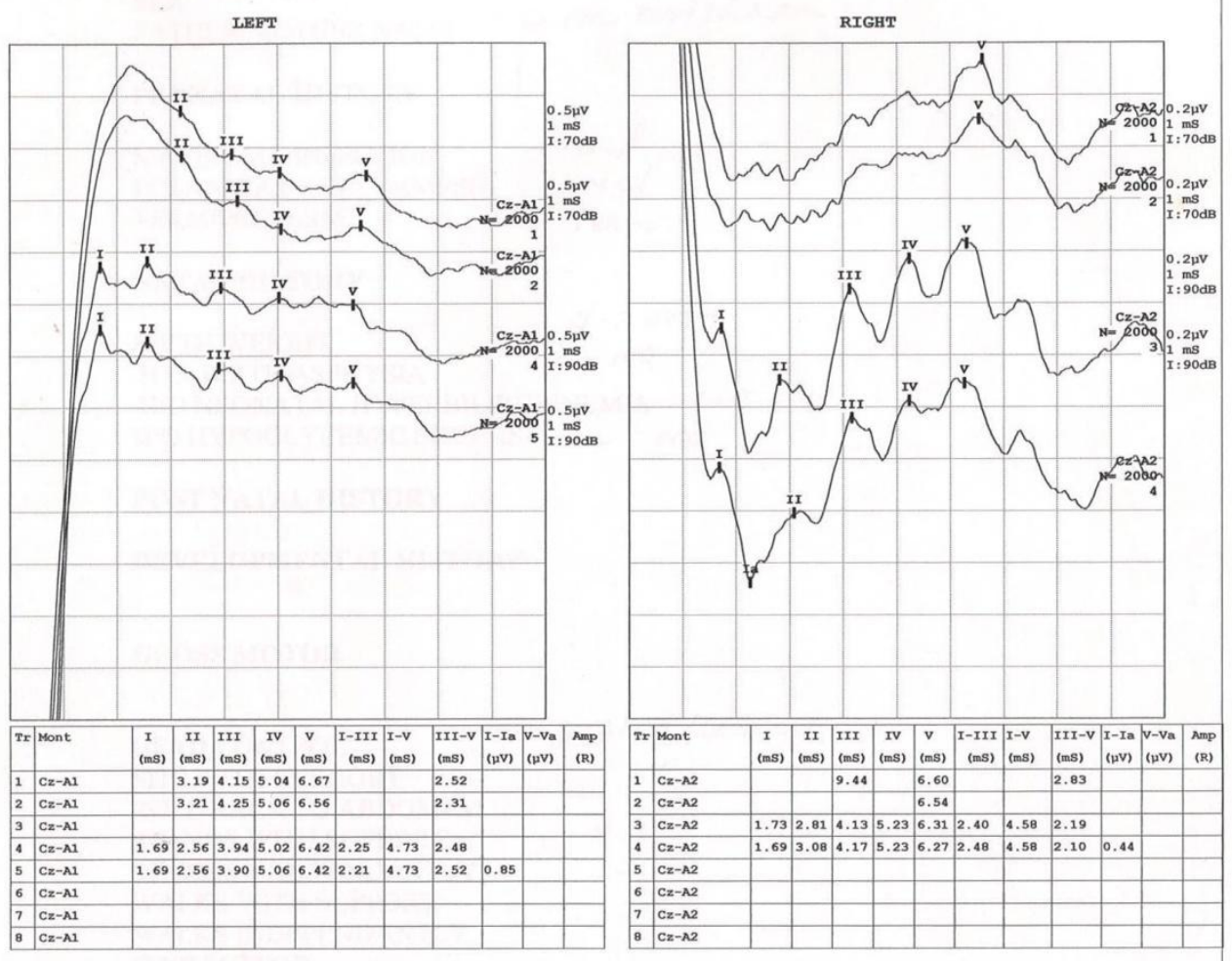

Fig 2. Absent BAEP trace

AEP RECORD ( $\mathrm{nHL}$ )
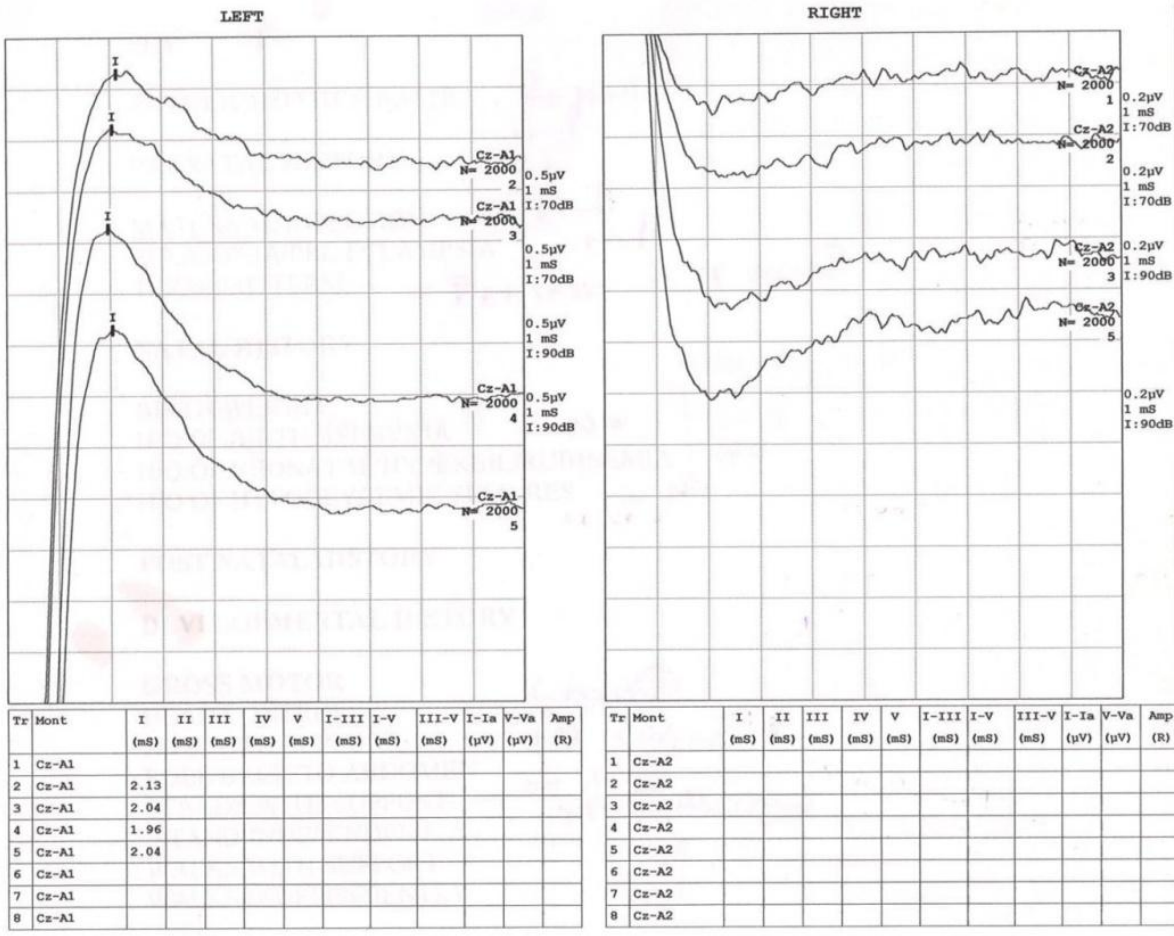


\section{Analysis}

Out of the $21 \mathrm{CP}$ children, mothers of 2 children had history of eclampsia. 5 children were preterm, 7 were having history of birth asphyxia \& 4 had neonatal hyperbilirubinemia.

At $70 \mathrm{Db}$ nhl $57.1 \%$ had normal BAEP. At $90 \mathrm{~dB}$ NHL $66.7 \%$ had normal BAEP. Of the 5 pretrm babies 4 had abnormal BAEP. Of the 16 term babies only 8 had abnormal BAEP at $70 \mathrm{~dB}$ NHL and 10 had abnormal BAEP at $90 \mathrm{~dB}$ NHL. Of the 14 children with h/o birth asphyxia 8 had abnormal BAEP AT $70 \mathrm{~dB}$ NHL and $10 \mathrm{had}$ abnormal BAEP at $90 \mathrm{~dB}$ NHL. All hypotonic children had abnormal BAEP. Of the 4 children with h/o neonatal hyperbilirubinemaia 3 had abnormal BAEP. Of the 7 children with grade 3 spasticity, only 2 had abnormal BAEP at $70 \mathrm{~dB}$ NHL and only 3 had abnormal BAEP at $90 \mathrm{~dB}$ NHL This may be due to the fact that in spastic $\mathrm{CP}$ the site of lesion may be in the cortex or basal ganglia. There was no relation between severity of clinical features \& BAEP abnormality. Even children with severe cerebral palsy had normal BAEP. The BAEP changes mentioned above were statistically not significant. (only selected tables are displayed here)

\section{Conclusion}

The BAEP abnormalities are more in children with h/o birth asphyxia, prematurity and neonatal hyperbilirubinemia. So all high risk babies should be screened with BAEP testing and if abnormality is present, should be followed up regularly and repeat BAEP done at 3 to 4 months interval. The BAEP can provide important information about a child's cochlear \& brainstem function. The results of BAEP testing should be interpreted cautiously. BAEP should not be considered in isolation. Although it can indicate dysfunction, it cannot determine the etiology.

\section{Limitations of study}

The small sample size may limit the usefulness of the data.

\section{References}

1. Kely M, Lubin RA,Keily JZ : Descriptive epidemiology of cerebral palsy. Publ Health Rev 1984.

2. Kudrajavcev T, Schoenberg BB, Kurland LT et al. Cerebral palsy trends in incidence and changes in concurrent neonatal mortality- Rochester, MN-Neurology 1983.

3. Bozynski $\mathrm{M}$, Nelson MGenace $\mathrm{K}$ et al. Cranial Ultrasoography and the production of cerebral palsy in infants weighing more than 1200 gms at birth. Dev Neurol 1988.

4. Kuban KC, Leviton A: Crerebral Palsy. N Engl J Med 1994.

5. Braddom 88888888888888

6. Eicher PS, Batshaw ML: Cerebral palsy, the child with developmental disabilities. Paedtr clin north Am 1993.

7. Hecox KE, Cone B Blaw ME (1981): Brain stem auditory evoked response in diagnosis of paediatric Neurologic diseases. Neurology

8. Majneber A, Rosenblatt B, Riley P (1988): Prognostic significance of BAEP in high risk neonates. Paed neurology 6: 367-374.

9. Auditory steady state response testing in children: evaluation of a new technology .Otolaryngol Head Neck Surg. 2003 Jul; 129(1):107-13.

10. Chadha S, Bais AS. Auditory brainstem responses in high risk and normal newborns. : Indan J Pediatr. 1997 NovDec; 64(6): 777-84.

11. Morlet T, Ferber-Viart C, Putet G, Sevin G, Duclaux R/ Auditory screening in high risk pre term and full term neonates using transient evoked otoacoustic emissions and brainstem auditory evoked otoacoustic emissions and brainstem auditory evoked potentials. Int J Pediatr Otorhinolaryngol. 1998 sep $15 ; 45(1): 31-40$.

12. Pasman JW, Rotteveel JJ, Maassen B,de Graaf R, Visco Y. Diagnostic and predictive value of auditory evoked 
responses in preterm infants: II. Auditory evoked responses. Pediatr Res. 1997 Nov; 42 (5):670-7.

13. Maison S, Duclaux R, Ferber-Viart C, Dubreuil C. Clinical interest of brainstem auditory evoked potentials in 72 children with inadequate language development. Int J Neurosci. 1996 Dec; 88(3-4):261-72.

14. Stueve MP, O'Rourke C. Estimation of hearing loss in children: comparison of auditoy steady state response, auditory brainstem response, and behavioural test methods. Am J Audiol. 2003 Dec; 12(2): 125-36.

15. Mjoen S, Langslet A, Tangsrud SE, Sundby A. Auditory brainstem responses (ABR) in high risk neonates. Acta Paediatr Scand. 1982 Sep;71(5):711-5.

16. Yamada $\mathrm{O}$, Ashikawa $\mathrm{H}$, Kodera $\mathrm{K}$, Yamane H. Frequency selective auditory brain stem response in newborns and infancts. Arch Otolaryngol. 1983 Feb; 109(2):79-82.

17. Tapia TocaMC, SavioLopez G. Stable auditory evoked potentials in the study of two patients with auditory neuropathy. Acta Otorrinolaringol Esp.2005 Jun-Jul; 56(6):240-5.

18. Luo R, Mai J, Chen Q, Yang S, Zhong J. Evaluation of sensorineural hearing loss in childhood. Zhonghua Er Bi Yan Hou Ke Za Zhi. 2001 Oct; 36(5):346-51.

19. Bao X, Wong Brainstem auditory evoked potential evaluation in children with meningitis. Pediatr Neurol. 1998 Aug; 19(2):109-12.

20. Mutch LW, Alberman E, Hagberg B, Kodama K, Velickovic MV. (1992) Cerebral palsy epidemiology: where are we now and where are we going? Dev Med Child Neurol 34: 547-555. 